\title{
La "etnicización" de la interculturalidad en el ámbito de la intervención socioeducativa. Las posiciones sociales del sujeto joven en contextos de diversidad cultural
}

\author{
Mara Gabrielli \\ Máster Interuniversitario Juventud y Sociedad, \\ Universitat de Girona \\ mara.gabrielli13@gmail.com
}

Resumen: En el caso de estudio, se analiza la gestión de la diversidad cultural en el ámbito de la intervención socioeducativa con jóvenes y los retos de las prácticas interculturales. En un contexto de "interculturalidad inducida" por la segregación espacial y social, la "agencia" del sujeto joven desarrolla itinerarios heterogéneos de (in)movilidad social. La pertenencia "étnico-cultural" sigue representando social y simbólicamente la posición social de las personas jóvenes y sus espacios de participación, lo que dificulta una relación de comunicación intercultural sustantiva sujeto-sujeto.

Palabras clave: jóvenes; diversidad cultural; intervención socioeducativa; educación no formal; competencias interculturales; empoderamiento.

Abstract: This study analyzes the management of cultural diversity in the socio-educational field with young people and the challenges of the intercultural practices. In a context of "interculturality induced" by the spatial and social segregation, the youth "agency" develops hetereogeneous itineraries of social (im)mobility. The "ethno-cultural" origin continues representing socially and symbolically the social position of young people and their spaces of participation, what hinders a relationship based on intercultural communication between subjects.

Keywords: youth; cultural diversity; socio-educational field; non-formal education; intercultural competences. 


\section{Introducción}

La hipótesis de partida de esta investigación sugiere que la construcción social y simbólica de una imagen problemática y conflictiva de la diversidad cultural dificulta la «agencia» de los sujetos jóvenes en igualdad de derechos y oportunidades. Desde el estudio de un caso concreto, se explora la gestión de la diversidad cultural en los espacios socioeducativos no formales como procesos que posibilitan o constriñen la autonomía y participación social de los sujetos jóvenes en contextos de diversidad cultural.

El presente artículo se estructura en tres bloques. Una primera parte en la que se hace una introducción conceptual al término interculturalidad y una breve contextualización con respecto a los retos de la educación intercultural en la intervención socioeducativa en España. Se delinean también los aspectos metodológicos de la investigación. En la segunda parte se exploran cómo el sujeto joven se relaciona con el contexto en el que vive (ámbito social, escolar y educación no formal), cuál es el planteamiento de los profesionales de la educación no formal hacia la diversidad cultural y la interculturalidad, y cómo se reflejan en la metodología y en la acción socioeducativa. En la última parte, desde un análisis cualitativo, se presentan los procesos de inserción en el campo social de cinco personas jóvenes que frecuentan el casal. ${ }^{1}$ En la síntesis conclusiva se hace una reflexión sobre los resultados más importantes, así como, sobre los posibles aportes futuros a la investigación en el ámbito de la educación intercultural.

Siendo esta una breve investigación de un máster de profesionalización en ámbito juvenil, no ha sido posible un análisis en relación a otros profesionales y agentes sociales (escuela, servicios sociales, familia, etc.), lo que sería de gran utilidad en futuras investigaciones para comprender de manera holística las posiciones sociales de los sujetos jóvenes en contextos de diversidad cultural.

1 En la obra de Bourdieu (1997) el «campo social» es un espacio social de acción y de influencia en el que confluyen relaciones sociales determinadas en la estructura de distribución de poder o capital. 


\section{Contextualización: los retos de la interculturalidad}

El cambio del mapa social de las sociedades europeas contemporáneas ha impulsado la implementación de la interculturalidad como modelo alternativo de "convivencia" entre culturas diversas. El concepto mismo de interculturalidad "supone una relación isométrica entre dos o más culturas, es decir, se relacionan y conviven en igualdad de condiciones, con respeto mutuo de sus formas de vivir y pensar" (Zárate, 2014: 94), pero la historia nos ha demostrado unas tendencias contrarias de "tolerancia" y de estrategias diversas de control ideológico del discurso y de la mente hacia otras culturas a fin de asimilarlas a los sistemas hegemónicos (Rodrigo, 2008; Zarate, 2014; Walsh 2010). Desde el pensamiento decolonial, Tubino (2005: 5-6) define estos procesos como "interculturalismo funcional" al sistema que invisibiliza "las crecientes asimetrías sociales, los grandes desniveles culturales internos y todos aquellos problemas que se derivan de una estructura económica y social que excluye sistemáticamente a los sectores subalternizados de nuestras sociedades." ${ }^{2}$ De acuerdo con estos pensamientos críticos, definimos como "etnicización de la interculturalidad" la vinculación de la diversidad cultural con el actual fenómeno de las migraciones transnacionales, aunque es tan solo uno de los aspectos que definen la complejidad misma de nuestra realidad.

En lo específico de la intervención social se ha puesto de manifiesto una cierta falta de sistematización y de fundamentación teórica en educación intercultural (Iglesias, 2014; Vilà, 2008) y la escasa integración de competencias interculturales para la comprensión de las diferencias culturales de los grupos minoritarios (Cohen-Emerique, 1999). En la actualidad española, Garreta (2011) destaca que la educación intercultural se ha ido fomentando más a nivel de discurso que de práctica.

Trilla (1993) define la educación no formal como toda actividad educativa en la que existe una intencionalidad educativa y una planificación de la experiencia de enseñanza-aprendizaje que acontece en aquellos contextos fuera de la educación reglada. En el caso de estudio, me refiero a un ámbito específico de la pedagogía social —la pedagogía del ocio- que Cuenca (2011) define

2 Esta teoría crítica contemporánea (también conocida como proyecto decolonial o como teoría posoccidental) se articula desde las ciencias sociales y humanidades de América Latina y Caribe como alternativa para reflexionar acerca de los debates sobre el colonialismo, la filosofía de la liberación y la pedagogía crítica, entre otras. 
como el campo teórico desde el cual abordar aquellas cuestiones vinculadas a las prácticas socioeducativas de la educación del ocio. En contextos de diversidad cultural, se presenta como un espacio importante para trabajar los aspectos vinculados a la convivencia en la diversidad mediante el aprendizaje de valores, actitudes y comportamientos hacia el reconocimiento y el respeto de la interculturalidad.

\subsection{Aspectos metodológicos de la investigación}

Este estudio se basa en una investigación realizada en un casal de joves (casa de jóvenes), entre marzo y mayo 2016, ubicado en el distrito V de Santa Coloma de Gramenet, una ciudad del área metropolitana de Barcelona que destaca por recibir a emigrantes, primero desde Andalucía, y después desde el flujo migratorio global. Según fuentes IDESCAT, en la década 2000-2010 se ha multiplicado por diez el número total de personas de nacionalidad extranjera censadas (un 23,8\% respecto al 2,3\% en el año 2000). ${ }^{3}$ Por distritos, el VI (Fondo) y el V (Santa Rosa, Raval y Safaretjos) son donde se concentra el mayor crecimiento de la población y donde se ubica la mayor población inmigrada en la ciudad: en el distrito VI casi la mitad de la población es de origen extranjero y en el distrito $\mathrm{V}$ este grupo llega a formar una tercera parte del total. ${ }^{4}$ Alrededor de 35 jóvenes de 16 a 21 años de los distritos Vy VI frecuentan con regularidad el centro y son jóvenes autóctonos y de origen extranjero (mayoritariamente marroquíes, pakistaníes, latinoamericanos y subsaharianos) que se encuentran en situación de riesgo social a causa de múltiples factores: desestructuración familiar, proceso migratorio, condiciones socioeconómicas precarias de las familias y escasos recursos de acceso a los estudios y al mundo laboral.

El casal pretende ofrecer a los sujetos jóvenes espacios de inclusión social que potencian su participación y autonomía en el contexto en el que viven. Desde una orientación metodológica integral, participativa y comunitaria, se desarrollan acciones de acompañamiento (tutorías periódicas y un plan de trabajo individualizado) para la (re)inserción en la educación formal y/o en el ámbito laboral. En situaciones más vulnerables se realizan también acciones con las familias, reuniones periódicas con la psicopedagoga del instituto (ubicado

3 Véase el siguiente enlace: > https://canaldidactico.wordpress.com/tag/santa-coloma-de-gramenet/>.

4 Véase el siguiente enlace: <https://canaldidactico.wordpress.com/2012/03/14/la-linea-9-de-metro-remodela-santa-coloma-de-gramenet/ $>$. 
en el mismo barrio del casal) y con profesionales de los servicios sociales. Con respecto a los recursos humanos, hay un único profesional (una educadora) contratado por un total de $30 \mathrm{~h} / \mathrm{semana}$, lo que dificulta el trabajo interno en términos de planificación y de evaluación del proyecto a medio y largo plazo. En relación a los recursos económicos, un aspecto interesante es la activación de becas de estudio financiadas por el proyecto europeo Garantía Juvenil con la finalidad de promover la (re)inserción de los jóvenes en la educación formal y posobligatoria.

Desde una perspectiva etnográfica, la intervención socioeducativa se ha analizado mediante la técnica de la observación participante durante las actividades (dentro del aula y en actividades de ocio fuera del centro). Con respecto al rol que se elige durante el trabajo de campo, se ha optado por un acercamiento abierto a los sujetos jóvenes comunicando el papel de la autora y el tipo de estudio que se estaba llevando a cabo. Esta técnica ha sido la base primaria de recogida de datos que ha permitido analizar las dinámicas internas del casal: en primer lugar, cómo los jóvenes socializan (con el grupo de iguales y con los profesionales/educadores), la autonomía personal y sus habilidades sociales; en segundo lugar, los planteamientos de los profesionales hacia la diversidad cultural y cómo se refleja en la promoción social de los sujetos jóvenes.

En un segundo momento, se han realizado entrevistas en profundidad (abril-mayo 2016) a dos profesionales del casal: una entrevista a la educadora del Proyecto Jóvenes del casal y a un educador que trabaja como formador en un curso de PFI y en el que participan algunos chicos y chicas del centro. En el mismo periodo, se han realizado también entrevistas en profundidad a cinco personas jóvenes usuarias del centro, como se detalla a continuación: 


\begin{tabular}{|l|l|c|c|c|c|c|}
\hline & $\begin{array}{c}\text { Nombre } \\
\text { ficticio }\end{array}$ & Sexo & Edad & $\begin{array}{c}\text { País de } \\
\text { procedencia }\end{array}$ & $\begin{array}{c}\text { Edad de } \\
\text { llegada }\end{array}$ & $\begin{array}{c}\text { Formación } \\
\text { académica }\end{array}$ \\
\hline 1 & Aimara & Femenino & 17 años & España & $\begin{array}{c}\text { Nacida en } \\
\text { España }\end{array}$ & PFI \\
\hline 3 & Julián & Masculino & 21 años & Chile & $\begin{array}{c}\text { A los cinco } \\
\text { años }\end{array}$ & Sin ESO \\
\hline 4 & Nadir & Masculino & 16 años & $\begin{array}{c}\text { Matrimonio } \\
\text { mixto: padre } \\
\text { marroquí, } \\
\text { madre } \\
\text { dominicana }\end{array}$ & $\begin{array}{c}\text { Nacida en } \\
\text { España }\end{array}$ & $\begin{array}{c}1 .^{\circ} \text { año } \\
\text { carrera } \\
\text { universitaria }\end{array}$ \\
\hline 5 & Santiago & Masculino & 16 años & $\begin{array}{c}\text { A los diez } \\
\text { años }\end{array}$ & $\begin{array}{c}1 .^{\circ} \\
\text { bachillerato }\end{array}$ \\
\hline Ecuatorial & $\begin{array}{c}\text { A los ocho } \\
\text { años }\end{array}$ & $\begin{array}{c}1 .^{\circ} \\
\text { bachillerato }\end{array}$ \\
\hline
\end{tabular}

La heterogeneidad de sus itinerarios se mide en función de la edad, la familia de origen (autóctona, biografía de inmigración; mononuclear, monoparental o matrimonio mixto), el recorrido académico (abandono escolar, [re]inserción en la educación formal e inserción en la formación posobligatoria) y, por último, los tipos de recursos socioeconómicos.

La realización de las entrevistas en el casal ha determinado un cierto condicionamiento espacio-temporal y, en algunos casos, la utilización de la grabadora ha creado situaciones de mayor cierre y dificultad de comunicación por parte de los sujetos jóvenes, sobre todo en relación al contexto familiar. En general, los padres son figuras poco visibles o ausentes en sus relatos, como he podido también constatar en mis propias notas de campo. Se ha obtenido información más específica a través de las reuniones y entrevistas a los profesionales del casal.

\section{La interculturalidad "inducida": un "orden socioespacial de marginalización"}

El casal objeto de este estudio está ubicado en un barrio de "exilio socioeconómico" y de "nueva pobreza y marginalidad urbana" retomando la definición del sociólogo francés Wacquant $(2007$ : 179) para describir aquellas zonas su- 
burbiales y obreras que condensan en su confinamiento territorial o espacial capas menos cualificadas de la clase obrera, altos índices de desempleo y precarización laboral, a lo que se añade la estigmatización social de barrios de "mala fama” en relación a los procesos de viejas y nuevas inmigraciones. Al llegar al barrio de Santa Rosa donde se ubica el centro, se percibe esa sensación de «condensación» socioeconómica y cultural de sus habitantes:

El Casal, un edificio nuevo frente al parque del motocross (como lo definen sus habitantes), está al lado de un instituto y de una escuela primaria en un área donde hay algunos pequeños comercios, la mayoría alquilados por personas de origen inmigrada. Destaca la presencia de población muy diversificada con respecto al origen sociocultural y que se hace particularmente visible a la hora de salida de la escuela en la mezcla de idiomas que se escuchan y que son el signo evidente de los cambios de la sociedad actual (autora, notas de campo).

En contextos de desventajas sociales, los jóvenes del casal perciben una mayor vulnerabilidad en barrios donde han aprendido a "ponerse capas", un chip que tienen activado para protegerse del mundo exterior, y donde han aprendido a construirse el personaje del "chulo que no tiene miedo":

Aquí en el barrio es una creencia que debes formar parte del grupo de los más chulos, de los chulitos del barrio, si no, te toca pringar. [...] Hay como una sensación de miedo, de necesidad, de protección para que los demás no te ataquen y así todo el mundo se construye un personaje que es chulo, un personaje que no tiene miedo (entrevista con una educadora del casal).

Es mentalidad. Tú no te lo planteas. Vas por la calle y de repente te viene uno que te empieza a provocar, te empieza a amenazar. Entonces, ¿qué haces? ¿Te quedas como un bobo y te vas corriendo? (Santiago, 16 años, origen: Guinea Ecuatorial).

En muchos casos son creencias populares, rumores, prejuicios alimentados por experiencias vividas de cerca o directamente, y también derivados de saberes implícitos. Las personas jóvenes aprenden a interiorizar el kit de duro de los padres y de su manera de relacionarse con el barrio:

Mi padre me dice: “№ hagas que te falten! ¡Defiéndete! Si te tocan, tocas” (Kevin, 16 años, origen ecuatoriano). 
Muchas veces se lo dicen a mi hermano, que no busque problemas, pero que si vienen a buscarle, por lo menos que plante cara (Anne, 18 años, origen catalán).

Una estigmatización social que se retroalimenta en el tiempo en la articulación de diversos campos sociales y simbólicos — una red de relaciones de acción y de influencia (Bourdieu, 1997) - que influye sobre la manera con la que los jóvenes construyen su relación con el contexto y entre el grupo de iguales. "Somos los malotes" es una expresión que los jóvenes utilizan a menudo visibilizando el estigma que reciben del exterior en relación también a situaciones vinculadas a pandillas locales y a las llamadas bandas latinas, según afirmaron algunos jóvenes durante un encuentro con los responsables del Proyecto Comunitario e Intercultural del Ayuntamiento de la ciudad. ${ }^{5}$ En general, se observa lo que describe Reguillo (2000: 107) con respecto a aquellas practicas sociales y culturales que no se conforman con la normatividad del mundo adulto e institucional: se "mira al joven como esencialmente contestatario y marginal", la calle asume un "papel de antagonista" que contrasta con los espacios escolares o familiares, y las "prácticas como el lenguaje, los rituales de consumo cultural, las marcas de vestuarios, al presentarse como diferentes", se perciben como "atentadoras del orden establecido". Así lo expresa Aimara durante la entrevista:

Uno también se cansa de decir que son los malos, todas las gentillas que llevan, así, gorras, y la gente se cree que son los típicos yonquis, que solo se dedican a fumar porros y no hacen nada, ¿sabes? Pero por ejemplo, a mi hermano le ha pasado muchas veces, y no. Los chavales, pobrecillos, también se cansan que se les diga esto.

Se observa que los jóvenes son conscientes del rol y de las posiciones sociales que se les atribuyen desde la familia, la escuela y, en general, desde el mundo adulto. Prevalece a menudo una visión adultocéntrica de la juventud como etapa de vulnerabilidad ante los factores de riesgo social, sin que se reconozca adecuadamente "su valor como sujetos de derechos y capital humano", de acuerdo con Krauskopf (2000: 126) ${ }^{6}$ Así lo expresa Julián durante la entrevista:

5 Giliberti (2013) destaca que en el imaginario social se consolida la relación entre bandas juveniles e inmigración latinoamericana en relación tanto a su estética como a un perfil sociocultural de clase baja.

6 Según esta autora, el desarrollo adolescente tiene que ver no solo con su biografía individual, sino también con la historia y el presente de su sociedad. 
Que los jóvenes son unos vagos, que no hacen nada, que siempre la están liando, que siempre están haciendo cosas que no deberían, por decirlo así, o que estén estudiando y que se pongan a trabajar. Ese prejuicio, que no hacen nada, que no valen nada, y eso es lo que intentamos cambiar con las actividades puntuales (Julián, 21 años, origen chileno).

Igualmente, la «etnificación» de las cuestiones juveniles (Queirolo, 2012), a mi parecer, refuerza la imagen de desviación y resistencia al orden establecido cuando la presencia del sujeto joven se visibiliza en los espacios públicos de las zonas urbanas, al mismo tiempo, invisibiliza la relación asimétrica de poder con la cultura dominante que quiere normativizar y controlar su presencia en los espacios sociales de las ciudades. Se destaca así lo que defino como una "interculturalidad inducida" por la segregación espacial y sociocultural a la que están sometidos los grupos sociales y étnicos desfavorecidos. Durante la entrevista, la educadora del casal lo expresa así:

Es como que las diferencias fueran marcadas por la clase social. Esa interculturalidad que ellos viven yo creo también que la marca la clase social. La viven de una manera muy natural, muy acertada, porque todos entienden que todos pasan por las mismas dificultades siendo de destinos diferentes. Quizá una clase social catalana alta juntada con una clase inmigrada con dificultades económicas, aquí se harían muchas diferencias raciales.

Desde el concepto de capital de Bourdieu (2000), en contextos de exclusión y marginalización, el capital económico y sociocultural de los grupos desfavorecidos es limitado y/o reducido debido a condiciones estructurales de desventaja social, y las formas con las que se desarrollan, adquieren y heredan estos mismos capitales por parte de las familias de estos jóvenes repercuten sobre sus itinerarios y trayectorias subjetivas. 


\subsection{Los mecanismos de "inclusión diferencial" en la educación formal ${ }^{7}$}

La escuela como espacio de socialización no es inmune a una representación problematizada de la diversidad cultural. En el contexto catalán, las intervenciones y medidas de "integración" han ido en dirección de procesos de "segregación intraescolar" a través de espacios educativos diferenciados destinados al "alumnado extranjero" o de agrupación por niveles (Carrasco et alii, 2011; Giliberti, 2013), así como expresa la educadora del casal en relación a la situación de algunos de los chicos que frecuentan el centro:

Tienen un aula de acogida todo el año [... . Pues claro, tú te sientes con competencias pero te tienen en una clase donde están los que son nuevos, los que tienen discapacidades o [...] los llaman el grupo C o el grupo D o algo así, que son los que tienen algún tipo de dificultad en el desarrollo, o los que están inmigrados y hablan poco de español. Se sienten, claro, en un grupo poco promocionador.

La educadora informa también de que estos jóvenes son percibidos como sujetos de difícil integración, potencialmente problemáticos, que se expresan con comportamientos disruptivos y actitudes de rebeldía. Según evidencian estudios anteriores, estas dinámicas justifican el poco interés por los estudios y una cierta resistencia hacia el aprendizaje bajo la idea de "profecía autocumplida” (Carrasco et alii, 2011) que vincula la diversidad étnico-nacional al nivel escolar del alumnado y que se basa en prejuicios culturales preconcebidos entre el profesorado (Gilberti, 2013). Bourdieu y Passeron (1977) informan de que los procesos de éxito y fracaso escolar esconden prácticas clasistas y racistas cuando el éxito se otorga en función del posicionamiento social de la familia de origen. ${ }^{9}$ Igualmente, Paul Willis (1988), en su estudio sobre los hijos de la clase

7 Siendo el ámbito escolar central para el desarrollo psicosocial y emocional de estos jóvenes, se aporta información también sobre las posiciones sociales del sujeto joven en el contexto escolar. En mis notas de campo, se observa una cierta dificultad por parte del joven a expresar abiertamente con el grupo de iguales sus malestares emocionales y las dificultades que puede experimentar en la escuela. En relación al contexto catalán, se recomiendan Garreta (2011) sobre la evolución de la educación intercultural, y Garreta y Palaudàries (2008) sobre la implementación de los planes de acogida.

8 El témino de "autoprofecia cumplida" fue acuñado por el sociólogo Robert K. Merton en su libro Teoría social y estructura social (1965).

9 Estos autores definen el capital como la acumulación de cultura propia de una clase que, heredada o adquirida a través de la socialización, más alta es la clase social de su portador y mayor es su peso en el mercado 
obrera de la escuela británica en los años setenta, destaca que la escuela aleja a los estudiantes socialmente subalternos de la educación formal hacia recorridos profesionales, contribuyendo a la reproducción de la clase social de la familia de origen. Así lo expresa la educadora del centro durante la entrevista:

El instituto parece estar en conflicto con el tipo de barrio. Quiere reivindicar la manera catalana, y no acepta la inmigración que tiene y la diversidad que tiene. [Y, por tanto, se activa lo que la educadora define como] una política de discriminación, de racismo abierto y de estigmatización según el origen de los chicos, y se les trata como un colectivo [... ] y los chicos se ponen en guerra.

En las reuniones de equipo y durante las entrevistas, los educadores del casal evidencian aquellos "mecanismos de etiquetaje" (Carrasco et alii, 2011) con los que se impulsa la orientación hacia trayectorias de formación profesional de los jóvenes de las clases sociales más bajas y de grupos "étnicos" que los alejan de la trayectoria académica ordinaria:

El profesorado para no enfrentarse a esa realidad deriva al joven a otro recurso peor, lo envía a un PFI, lo envía a una UEC [...]. Simplemente, se valora los que no llegan para enviarles a otro recurso. Claro, eso te deja etiquetas como chico [... ]. Hoy en día si no tienes la ESO es un indicador de exclusión social casi absoluto (entrevista con la educadora del casal).

La mateixa escola fa temps li va fer una mica el buit a l'alumne i que no conta amb ell per poder desenvolupar itineraris més promocionadors [...]. Llavors des d'un primer moment en l'ESO s'ls comença a comentar la possibilitat d'anar a un PFI [... ]. Això comporta què molts d'aquests alumnes tinguin una autoestima molt fluixa, que desenvolupen moltes dinàmiques de carrer, $[\ldots]$. Llavors, tenim un perfil d'alumnes [...] que es troben en situacions de immobilitat perquè és com si constantment estiguessin intentant demostrar que ells no serveixen i llavors no es comprometen (entrevista con educador, curso PFI, casal joves).

Estos mecanismos de "inclusión diferencial" (Mezzadra, 2007) determinan situaciones de baja autoestima, dificultades emocionales, sentimientos de re-

simbólico cultural. Bourdieu (2000) distingue entre el "capital económico" (el poder político y hegemónico mediante el control sobre los recursos económicos), el "capital social" ("un capital de obligaciones y relaciones sociales» de las redes de influencias del grupo social), el "capital cultural" (conocimiento, educación, habilidades y ventajas transmitas), y el "capital simbólico" (el prestigio acumulado o adquirido mediante el reconocimiento de los agentes del campo), que consiste en una serie de propiedades intangibles inherentes al sujeto. 
chazo hacia la institución escolar que — en palabras de la educadora - crea "desobedientes sociales" que buscan otras estrategias de afirmación y reconocimiento dentro y fuera de la escuela. Como evidencian Carrasco et alii (2011) y Giliberti (2013), son estos mecanismos de contracultura escolar y desafiliación los que reafirman la profecía autocumplida del profesorado:

Todos han ido adoptando su rol en el que los educadores son malos, van tomando el rol de carceleros y los chavales hacen guerra contra ellos. Y se genera la dinámica en la que los jóvenes son los encarcelados, e intentan ir en contra de los funcionarios (entrevista con educadora del casal).

Según lo expresado por la educadora del casal, estos enfoques pedagógicos no tienen en cuenta los aspectos relacionales y emocionales en el proceso de incorporación al sistema educativo, a falta también de un soporte mayor a la condición social de estos jóvenes. Jordán $(2002,2007)$ informa de que el profesorado tiende a primar los aspectos lingüísticos-instrumentales sin cultivar apenas la dimensión "afectiva-actitudinal-moral”. Según destacan Garreta (2011) y Jordán (2007), en la práctica, el discurso intercultural no ha conseguido alejarse de una cierta orientación asimilacionista y segregacionista. Por ende, toma relevancia el tipo de enfoque que se le da a los discursos y a las praxis educativas en definir la inclusión social de los sujetos jóvenes desde la pluralidad de sus subjetividades.

\subsection{La "etnicización" del discurso sobre interculturalidad desde la intervención socioeducativa}

Desde una perspectiva pedagógica y psicopedagógica, las competencias interculturales abordan cuestiones comunicativas, comprensivas y transformadoras desde el ámbito cognitivo, afectivo y comportamental (Iglesias, 2014; Vilà, 2008), que ayudan a visibilizar la relación de poder mediante la toma de conciencia sobre nuestras propias cosmovisiones como base para la comprensión de las diferencias culturales. ${ }^{10}$ Según evidencia Iglesias (2014), también en el caso de estudio se observa que, sin una formación adecuada, y sin un plantea-

10 La dimensión cognitiva permite una primera toma de conciencia de los propios bagajes culturales y de los demás mediante una acción (auto)reflexiva con la que adoptar competencias y estrategias hacia interacciones eficaces en los procesos comunicativos y relacionales. La dimensión afectiva supone trabajar emociones que sean positivas para comprender la realidad cultural. La dimensión comportamental supone la materialización de habilidades comunicativas y transversales (Iglesias, 2014; Vilà, 2008). 
miento consciente o ideológico, los profesionales del casal proyectan visiones y actitudes esencialistas y culturistas cuando se confunde la igualdad (entendida como el recibir el mismo trato para evitar desigualdades) y la equidad (como similitud entre los sujetos), que se traduce en actitudes o acciones asimilacionistas, y cuando se enfatizan las diferencias desde una perspectiva multicultural que explica todo tipo de actitudes y comportamientos.

Desde una aproximación teórica al método de «choque cultural», ${ }^{11}$ un modelo desarrollado por Cohen-Emerique (1999), psicóloga experta en relación y comunicación intercultural, se analiza el discurso y planteamiento sobre diversidad cultural e intercultural por parte de los profesionales del casal. ${ }^{12}$ CohenEmerique (1999: 466) destaca que los prejuicios, el etnocentrismo y la tendencia a esquematizar las diferencias constituyen «filtros y pantallas conectivas y afectivas que obstaculizan el desarrollo de una abertura a la cultura del otro», generando así situaciones de "choque cultural" como "reacción de extrañamiento" (sentimiento de rechazo, ansiedad, hasta el asombro positivo). En relación a la socialización y a la gestión de los espacios públicos, se percibe una dimensión colectiva de control y regulación simbólica de los roles sociales que contrasta con los procesos de individualización de las relaciones sociales y familiares en las sociedades occidentales. Durante la entrevista, en relación a la socialización de los jóvenes, la educadora del centro comenta:

Aquí todo funciona por clanes, porque, en realidad, los marroquíes son un tipo de cultura que, más las madres [... ], viven en comunidad. Los de Bangladesh, los pakistaníes, tienen una comunidad también, y los chinos tienen una comunidad también muy grande aquí, pero no en el casal, en el barrio. Se dan mucho apoyo entre ellos, son comunidades. De los padres viene la necesidad de formar parte de un grupo colectivo. [...] Pero creo que el catalán está más libre de eso porque quizá aquí cada uno vive en su piso, más occidental, más

11 El término «choque cultural» fue introducido por primera vez en 1951 por la antropóloga estadounidense Cora DuBois para referirse al sentido de desorientación y esmarrimiento que experimentaban los antropólogos en contacto con culturas diferentes. Por tanto, se ha asumido en relación a las dificultades que pueden encontrar las personas a nivel interpersonal con otras culturas.

12 Esta autora ha revisitado el método llamado «Análisis de Incidentes Críticos» para adaptarlo a los profesionales que trabajan en el ámbito de la interculturalidad. En su forma actual fue elaborado por el coronel John C. Flanagan como parte del Programa de Psicología en Aviación de las Fuerzas Aéreas del Ejército de Estados Unidos durante la Segunda Guerra Mundial. Desde entonces este método se ha utilizado para determinar las competencias de profesionales de diferentes disciplinas. (Véase: Flanagan, John C. Psychological Bulletin, vol. 51, n. ${ }^{\circ} 4$, julio 1954). 
independiente, no nos apetece saludar al vecino mientras que ellos valoran esa realidad, conocerse entre ellos. El barrio está formado por un montón de colectivos, más cerrados o menos cerrados, pero colectivos, no individuos.

En el mundo occidental la noción de persona está relacionada con normas que valorizan su autonomía e independencia, mientras que en las sociedades donde la comunidad tiene mayor peso, prima el grupo sobre el individuo, aunque un cierto tipo de relaciones sociales más codificadas corresponden a determinadas clases sociales, según evidencia Cohen-Emerique (1999). En el discurso de los profesionales del casal se asume la diferencia cultural como factor obstaculizador de la comunicación. Los comportamientos "culturales" de los padres se perciben así como portadores de una cultura "étnico-cultural" fija y monolítica que se resiste al cambio, frente a los hijos que representan un cambio de modelo que favorece su reconocimiento en la sociedad de recepción. En relación a la situación familiar de uno de los chicos pakistaníes del centro, durante una charla informal, la educadora afirma:

Él vino a España a los 9 años y se integró totalmente en el contexto español, [mientras que] el padre es muy conservador, rígido y muy religioso. [Lo que según ella dificulta la relación familiar entre ellos porque el joven] es el español de la situación que igual no pinta nada con el resto de la familia $[y]$ no quiere relacionarse con chicas pakistaníes ni casarse con ninguna de ellas.

Igualmente, se tiende a explicar actitudes y comportamientos de los jóvenes pakistaníes mediante expresiones que los definen como personas "más calladas, más reservadas, que les tienen mucho respeto a los adultos" a causa de contextos familiares donde los padres ejercen «un control extremo sobre los hijos», son "muy conservadores" y "muy religiosos". En general, se perciben como jóvenes que quedan "más invisibilizados", y su supuesta menor proximidad sociocultural a la cultura de recepción se percibe a menudo como un obstáculo que dificulta la intervención socioeducativa sin que esto se corresponda con un cambio metodológico que favorezca su mayor implicación o participación en las actividades del centro.

En la cultura pakistaní no es el padre el que manda, el que manda es el abuelo o el que haga de patriarca, puede ser el hermano, puede ser el padre. En Pakistán el patriarca manda hasta en las familias que emigran. Al final, si mi 
familia es digna o no es digna pasa por los ojos del patriarca (entrevista con educadora del casal).

También se destaca una percepción de lo tradicional como "déficit cultural del otro" - implícitamente del mundo musulmán - como un factor que obstaculiza el aprendizaje educativo de estos jóvenes. Según evidencia también Vilà (2008), se muestra una tendencia a simplificar las diferencias mediante filtros cognitivos y afectivos desde los códigos culturales del profesional que obstaculizan la comprensión de aquellos "sesgos culturales" en términos de enseñanza y aprendizaje que pueden variar entre las culturas.

Clar que hi ha uns tipus de competències i de formació bàsica que nanos nouvinguts no tenen $[\ldots]$, tenen uns tipus de handicaps en relació a altres nanos que han estat educats aquí. El tema cultural d'alguna manera per com està la infraestructura cultural en tema d'educació en el país d'origen, d'un poble o no, són mancances pel que fa a l'aprenentatge que continuen tenint aquí. Són buits (educador, curso PFI, casal joves).

Igualmente, las relaciones de género entre hombres y mujeres generan una reacción de mayor indignación por parte de las profesionales, aunque es una visión compartida por los profesionales varones de la entidad:

Una hija pakistaní es una molestia hasta que la casen a los 15 o 16 años, ya la tienes parada porque te cuesta tener una hija. El hijo te da dinero, trabaja y se casará con otra familia que juntará. En cambio, las chicas son para trabajar en la casa y hay que colocarlas con un hombre aquí, en Barcelona (entrevista con educadora del casal).

Després en preceptes o valors també com a societat tenen uns tipus de característiques que als joves d'altres cultures sobretot de cultures musulmanes els costa molt assolir, identificar i respectar o no, el tema d'identitat de genere, per exemple (entrevista con educador, curso PFI, casal joves).

Según Cohen-Emerique (1999: 482), esta percepción "choca contra las representaciones, imágenes, aspiraciones, que estas profesionales europeas tienen respecto a sí mismas", en comparación con los cambios sociales en relación al estatus de la mujer en la sociedad occidental, lo que genera a menudo un deseo de transformación social vinculado a sus propias posiciones ideológicas conflictivas a nivel intra o interindividual. Considero que son percepciones sesgadas 
que dejan de lado las estructuras sociales y socioeconómicas que están en la base de la conformación de identidades colectivas e individuales dentro de contextos geopolíticos e históricos heterogéneos y plurales.

\section{Distintos procesos biográficos de inserción en el campo social}

En el análisis de los procesos de autonomía y promoción social de los cinco casos de estudio, se retoma el modelo de "asimilación segmentada" formulado por Portes y Fernández-Kelly (2006) y reelaborado por Romaní et alii (2012). ${ }^{13} \mathrm{Se}$ prefiere hablar de "inserción segmentada" por la estrecha relación del concepto de "asimilación" - y también de "integración" - al contexto de llegada como una condición unidireccional de inclusión a la sociedad mayoritaria que no deja espacio para una inserción híbrida, dinámica, que reformula constantemente su adaptación y que no reclama ninguna inserción total. ${ }^{14}$ Asimismo, en el análisis de la "agencia personal" se analizan algunos indicadores elaborados por Jennings et alii (2006), Pick et alii (2007) y Morton y Montgomery (2013). ${ }^{15}$

En los cincos casos estudiados, se evidencian situaciones de "movilidad ascendente", de "inserción selectiva" y de "(in)movilidad o movilidad descendente" en las que las características familiares influyen mayormente en los itinerarios biográficos de estos jóvenes. De acuerdo con Rodrigo y Medina (2008), se evidencia que la biografía de migración familiar puede tener un peso más o menos traumático según el sentido que las personas jóvenes le confieren al “origen”, así como en relación al tipo de inserción familiar en la sociedad de recepción, sus dinámicas internas de adaptación al contexto social y sus condiciones socioeco-

13 Con el concepto de "asimilación segmentada", Portes et alii (2006) se refieren a un conjunto de resultados estratégicos que no asumen una trayectoria uniforme de asimilación sino de divergencia. Mientras, Romaní et alii (2012) proponen tres modelos: "asimilación selectiva" (selective acculturation), en la que los vínculos comunitarios son claves para la inclusión social; un modelo de "plena asimilación" (full assimilation); y una "asimilación descendente o de etnicidad reactiva" (the descendent and/or reactive etbnicity assimilation) que supone una asimilación a las clases más bajas y de permanente pobreza.

14 De acuerdo con Queirolo (2012: 23), los conceptos de "asimilación" y de "integración" son términos ambiguos y polisémicos vinculados a elementos socioeconómicos y culturales, y son funcionales a las lógicas de la sociedad receptora que a menudo «turns back on the individual's capacity to integrate better».

15 Pick et alii (2007) definen la «agencia personal» como un proceso psicosocial interno que se empodera en el contexto exterior cuando adquiere una conciencia crítica sobre su realidad sociopolítica y económica. 
nómicas. ${ }^{16}$ En el caso de familias más estructuradas, cuando el control parental se da en condiciones de soporte, apoyo emocional y desde una relación basada en el respeto mutuo de las diferencias internas, los sujetos jóvenes tienden a una "movilidad ascendente" y de "inserción selectiva" que influye positivamente hacia el éxito académico y sus expectativas.

Namir, familia súper estructurada [...]. Hijos muy queridos, muy buscados, muy respetados por los padres [... ], y eso les ayuda mucho a poder tomar su camino con relativa tranquilidad (entrevista con educadora del casal).

En mi casa hay dos culturas, la marroquina y la dominicana. Bueno, diferencias hay, pero son diferencias mínimas, es decir, ya que mis padres no siguen ninguna religión más respetable, y eso facilita la convivencia (entrevista con Maribel, 18 años).

En relación a la escala ESAGE elaborada por Pick et alii (2007) ${ }^{17}$ estos jóvenes muestran la habilidad de definir de manera autónoma sus propias metas y objetivos y de tener control sobre sus elecciones y decisiones en términos de autoeficacia (un cierto grado de control sobre sus pensamientos, emociones y acciones), capacidad de autonomía (como estado de autogobierno interno a la persona sin control externo que lo delimita), capacidad de autodeterminación (en términos de motivación personal en actividades de su interés y satisfacción espontánea) y, por último, capacidad de autorregulación y control sobre sus conductas y sobre su entorno. ${ }^{18}$ Igualmente, el soporte socioemocional y económico del casal es un factor que incide positivamente hacia el empoderamiento del sujeto joven, también en condiciones de mayor desestructuración familiar y de escaso capital sociocultural de la familia.

Aimara también viene de una situación monoparental [...]. Dejó la ESO cuando murió su abuela [...]. Ahora está haciendo un proceso para reengancharse al proceso de educación formal con un PFI de electricidad [... ], y este

16 Se prefiere no utilizar el concepto de "segunda generación" de acuerdo con lo que expresa Queirolo (2012: 24): "The category second generation, placing an emphasis on the conservation of the cultural distance, reminds the children of immigrant families and popular classes that, in spite of their efforts, they will always be tied to another cultural space, and therefore, their existence does not deserve full citizenship".

17 Escala Para Medir la Agencia Personal y el Empoderamiento.

$18 \mathrm{La}$ autoestima y la autoeficacia se consideran como los indicadores principales del cambio psicosocial de los sujetos jóvenes (Morton y Montgomery, 2013). La autoeficacia — concepto introducido por el psicólogo Bandura (Pick et alii, 2007) - indica la creencia de la persona en sus propias habilidades y juega un rol importante con respecto a los objetivos y a las metas que se prefije conseguir. 
año empezará un grado medio de actividad físico-deportiva en medio natural. Hemos conseguido una beca en la Escuela Pía de Sarriá para que cambie de barrio, para que tenga otros impulsos en su vida, otras relaciones (entrevista con educadora del casal).

En el caso de procesos migratorios de exclusión y/o de inclusión negativa, los itinerarios biográficos de estos jóvenes tienden a situaciones de "(in)movilidad o de movilidad descendente" en función también de las condiciones socioeconómicas precarias de la familia y una cierta movilidad de su estructura interna (ausencia de referentes masculinos, segundo matrimonio y relación conflictiva con el padrastro).

Julián. Es un chico chileno y se vino aquí con cinco años. Lleva 16 años aquí, en España. Tiene 21 años ahora. La madre tuvo a Javier y se separó del padre. Con este padre hubo muchos problemas, muchos conflictos en tema de malos tratos. [... Estudió la ESO y no la acabó. Hizo un PFI y lo dejó. [...] No tiene permiso de residencia ni de trabajo [...]. Está como en un limbo. [...] No consigue asumir sus responsabilidades. [... ] Hay un tema de autoestima, de muy baja creencia en sí. Pone a los demás por delante (entrevista con educadora de casal).

En términos de autoeficacia (Morton y Montgomery, 2013), las condiciones sociojurídicas de exclusión debilitan tanto la capacidad del sujeto joven de tomar decisiones y asumir sus propias responsabilidades como el autorreconocimiento personal de las propias potencialidades y habilidades ante el miedo al fracaso o al rechazo del contexto social. Igualmente, según lo expresado por Rodrigo y Medina (2008), se puede afirmar que la situación social de "limbo" refuerza el sentimiento de pertenencia a un pasado como "raíz", es decir, como algo idealizado e inamovible y como marco de referencia en la sociedad de recepción siendo la red coétnica familiar el único ámbito en el que el joven se siente aceptado y reconocido como miembro del grupo. 
De aquí totalmente no me siento. Me siento más chileno [...], me siento como si hubiera estado toda mi vida en Chile, me sale todo el acento, todo lo que sea, madre mía. Esto no soy yo, dónde estoy, esto no soy yo.

Cuando la familia ejerce una tutela de sobreprotección y de infantilización de la posición del hijo en el ámbito familiar, sin el apoyo socioemocial que este requiere, el joven vive estas situaciones como opresión familiar y la relación se vuelve conflictiva. Esto puede derivar en lo que Rodrigo y Medina (2008: 157) definen como "amputación psicológica" con el pasado familiar, lo que determina un proceso de inclusión total en el contexto de recepción.

Santiago es un chico de Guinea Ecuatorial. [... ] Es un chico muy inteligente, [... ] y vive en un entorno en su casa donde todo el día le están diciendo que hace mal las cosas [...]. Vive muy oprimido (entrevista con educadora de casal).

Cada uno aquí pasa cosas en su familia, lo que sea, y a algunos les ha traumatizado y a otros les ha frenado, ¿sabes? [... Entonces, aquí, en el casal, lo que quería primero [era] empezar a abrirme, empezar a tener contactos con personas, y después ya mirar los fallos (entrevista con Santiago, 16 años).

De acuerdo con Romaní et alii (2012), la movilidad de estos jóvenes se da en función de su posición en la sociedad de recepción, y de su trayectoria hacia la vida adulta: un "doble viaje" (dual journey) que intensifica su vulnerabilidad como generación, y su inclusión y/o exclusión está vinculada al concepto mismo de ciudadanía, no solo en términos jurídicos sino más bien como fenómeno sociológico y político, que implica una relación mucho más compleja del individuo con la estructura sociopolítica de la sociedad dominante.

\section{Síntesis conclusiva}

En este estudio se evidencia una situación de "interculturalidad inducida" por la segregación espacial y sociocultural a la que están sometidos los grupos más desfavorecidos y las minorías étnicas en la articulación de nuevas formas de estratificación y desigualdades sociales. El "estigma étnico" y "cultural" sigue representando social y simbólicamente la posición social y los espacios de parti- 
cipación de los sujetos jóvenes en contextos de diversidad cultural cuando se mira a la interculturalidad como un "fenómeno social" acotado en un espacio sociotemporal concreto en la relación sujeto-sujeto, obviando así todo proceso dinámico inherente a los cambios sociales de nuestras sociedades urbanas.

Se puede también afirmar que el mayor reconocimiento positivo de la diversidad del «otro» se da cuanto mayor es la proximidad sociocultural de origen del joven obviando una realidad mucho más compleja tanto en términos de mecanismos psicosociales complejos así como con respecto a dinámicas de «asimilación» y diferenciación subjetivas y heterogéneas. El proceso de inserción en la sociedad de instalación implica siempre un cierto grado de (re)apropiación subjetiva de códigos y valores socioculturales, tanto por parte de las familias como de sus hijos. En los cinco casos de estudio, se observa una «inserción segmentada» en el campo social que evidencia su heterogeneidad y dinamismo interno en relación con la estructura familiar (su capital humano y cultural), su capital social y económico (su posición y condición social), y el rol que el joven adquiere en la sociedad de recepción.

Se reclama así una intervención socioeducativa dirigida a la educación intercultural con los sujetos jóvenes, y también mediante planes de formación en competencias interculturales para los profesionales de la intervención social, así como una mayor interconexión entre el ámbito formal y no formal de la educación. Solo de esta manera la acción socioeducativa tendrá una incidencia transformadora en la realidad social en la que se adscribe.

Quisiéramos aquí concluir con aquello que expresa Tubino (2005: 3) respecto al concepto de interculturalidad y que, en mi opinión, evidencia una situación presente que necesita una reformulación epistémica, ética, política y pedagógica hacia la "interculturalidad crítica" (Walsh, 2010) asumida como proceso inherente a nuestra compleja realidad social:"La interculturalidad no es un concepto, es una manera de comportarse. No es una categoría teórica, es una propuesta ética. Más que una idea es una actitud, una manera de ser necesaria en un mundo paradójicamente cada vez más interconectado tecnológicamente y al mismo tiempo más incomunicado interculturalmente". 


\section{Algunas consideraciones finales}

Durante el desarrollo de esta breve investigación me he enfrentado a algunas dificultades que, en cierta medida, limitan los resultados. En este sentido, el tiempo ha supuesto el principal obstáculo, obligándome a acotar la muestra objeto de investigación y a limitar también los resultados con respecto al entorno social y al ámbito de la educación formal. Sin embargo, los objetivos iniciales podrían considerarse confirmados en relación a los resultados obtenidos. Por ende, este estudio supone solo un primer paso para futuras investigaciones. Se considera la posibilidad de abarcar un análisis cualitativo de mayor impacto a través de historias de vida en las que se pueda explorar la posición social de los sujetos jóvenes tanto en el ámbito formal como no formal de la educación en contextos de diversidad cultural. 


\section{Bibliografía}

Bourdieu, P. y Passeron, J. C. (1977). La reproducción. Elementos para una teoría del sistema de enseñanza. Barcelona: Editorial Laia.

Bourdieu, P. (1997). Razones prácticas. Sobre la teoría de la acción. Barcelona: Anagrama.

Bourdieu, P. (2000). Poder, derecho y clases sociales. Desclée de Brouwer.

Carrasco, S.; Pàmies, J.; Ponderada, M.; Ballestín, B. y Bertrán, M. (2011). "Segregación escolar e inmigración en Cataluña: aproximaciones etnográficas”. En: García Castaño, F. J. y Carrasco, S. (eds.). Investigaciones en Inmigración y Educación en España. Homenaje a Eduardo Terrén Lalana. Madrid: CIDE-Ministerio de Educación.

Cohen-Emerique, M. (1999). "Análisis de incidentes críticos: un modelo para la comunicación intercultural”. Revista Antipodes, 145: 465-480.

Cuenca Cabeza, M. (2011). "El ocio como ámbito de Educación Social”. Educación Social, (47): 25-40.

Garreta, J.(2011). "La atención a la diversidad cultural en Cataluña: exclusión, segregación e interculturalidad”. Revista de Educación, 355(mayo-agosto): 213-233.

Garreta J. y Palaudàries J. M. (2008)."La acogida del alumnado de origen inmigrante: un análisis comparado desde la situación en Cataluña”. Revista Española de Educación Comparada, (14): 49-78.

Giliberti, L. (2013). "La condición inmigrante y la negritud en la experiencia escolar de la juventud dominicana: estigmas y formas de agencia. Una etnografía transnacional entre la periferia de Barcelona y Santo Domingo". [Tesis con mención de Doctorado Internacional]. Universidad de Lleida, Departamento de Geografía y Sociología.

Iglesias Vidal, E. (2014). "La formación en interculturalidad en la educación en el tiempo libre: necesidades y estrategias desde una perspectiva de competencias interculturales". Revista Electrónica Interuniversitaria de Formación del Profesorado, 17(2): 167-182.

Jordán, J. A. (2002). "Educación intercultural y sociedad plural”. Teoría de la Educación, (14): 93-119.

Jordán, J. A. (2007). “Formación intercultural del profesorado de secundaria”. Estudios sobre Educación, (12): 59-80. 
Krauskopf, D. (2000). "Dimensiones críticas en la participación social de las juventudes”. En: Participación Social y Política de los jóvenes en el horizonte del nuevo siglo. Buenos Aires: Edición Florencia Enghel. CLACSO.

Mezzadra, S. (2007). "Confini, migrazioni, cittadinanza”. Papers: revista de sociologia, $(85): 31-41$.

Queirolo Palmas, L. (2012). "The social construction of the youthmigration nexus in contemporary Spain. A critical overview". Italian Journal of Sociology of Education, (3): 3-33.

Reguillo, R. (2000). Emergencia de culturas juveniles. Buenos Aires: Norma.

Rodrigo, M. y Medina, P. (2008). "Juventud y comunicación: el impacto de los medios de comunicación en la transmisión de valores interculturales. Juventud y diálogo entre civilizaciones”. Revista de Estudios de Juventud, (80): 151-157.

Romaní, O;; Feixa, C. y Latorre, A. (2012). "Being Heard or Being Seen. To participate or not to participate”. En: FAngen, K. et alii (eds.), Young Adult Migrants Lives in Seven European Cities. London: Palgrave-MacMillan, pp. 146-172.

Trilla, J. (1993). La educación fuera de la escuela: ámbitos no formales y educación social. Barcelona: Ariel.

Tubino, F. (2005). La interculturalidad crítica como proyecto ético-político. Encuentro continental de educadores agustinos, del 24 al 28 de enero de 2005. Disponible en: <https://oala.villanova.edu/congresos/educacion/lima-ponen-02.html> [Consulta 30/03/2016].

VIlÀ BAÑos, R. (2008). “Cómo educar en competencias interculturales? Una alternativa a la educación formal”. Revista de Estudios de Juventud, 80: 77-93.

Wacquant, L. (2007). "Marginalidad urbana en el próximo milenio". En: Parias Urbanos: Marginalidad en la ciudad a comienzo del milenio. Buenos Aires: Manantial, pp. 163-188.

Walsh, C. (2010). "Interculturalidad crítica y educación intercultural”. En: Viaña, J; Tapia, L. y Walsh, C. Construyendo Interculturalidad Crítica. Instituto Internacional de Integración del Convenio Andrés Bello, IIICAB, pp. 75-96.

Willis, P. ([1988] 1977). Aprendiendo a trabajar. Cómo los chicos de clase obrera consiguen trabajos de clase obrera. Madrid: Akal.

Zárate Pérez, A. (2014). "Interculturalidad y decolonialidad". Tabula Rasa, (20): 91-107. 